\title{
Constrained Variation in Jastrow Method at High Density
}

\author{
J. C. OWEN \\ Department of Theoretical Physics, \\ University of Manchester, Manchester M13 9PL, England
}

\begin{abstract}
AND
R. F. BISHOP AND J. M. IRVINE

Department of Theoretical Physics, University of Manchester, Manchester M13 9PL, England, and Daresbury Laboratory, Daresbury, Warrington WA4 4AD, England
\end{abstract}

Received July 8, 1975

\begin{abstract}
A method is derived for constraining the correlation function in a Jastrow variational calculation which permits the truncation of the cluster expansion after two-body terms, and which permits exact minimization of the two-body cluster by functional variation. This method is compared with one previously proposed by Pandharipande and is found to be superior both theoretically and practically. The method is tested both on liquid ${ }^{3} \mathrm{He}$ using the Lennard-Jones potential and on the model system of neutrons treated as Boltzmann particles ("homework" problem). Good agreement is found both with experiment and with other calculations involving the explicit evaluation of higher-order terms in the cluster expansion. The method is then applied to a more realistic model of a neutron gas up to a density of 4 neutrons per $F^{3}$, and is found to give ground-state energies considerably lower than those of Pandharipande.
\end{abstract}

\section{INTRODUCTION}

The desire to study dense neutron matter (density $>10^{14} \mathrm{~g} \mathrm{~cm}^{-3}$ ) and the problem of its possible solidification has produced a new interest in variational techniques, particularly the method of Jastrow correlation functions [1,2]. The hope is that such techniques may prove more appropriate for microscopic calculations at such extreme densities than the already well-advanced methods of perturbation theory. The difficulty with the Jastrow method arises through the calculation of the many-body $(\geqslant 3)$ terms in the cluster expansion, and it is well known that some allowance must be made for these higher-order terms, either by explicit 
calculation or by constraining the correlation function in some way, in order to obtain sensible results [3].

Several years ago, Pandharipande advanced a method of lowest-order constrained variation (L.O.C.V.) [4], where the correlation function was forced to heal rapidly and was put equal to unity beyond some healing distance, $d$. This was not a purely variational method, however, since it required the addition of a constant parameter, $\lambda$, to the interparticle potential, which was justified by analogy with Brueckner perturbation theory [5] and by purely physical arguments. When applied to a hypothetical model system of neutrons treated as Boltzmann particles [6] it gave positive values for the energy per particle which were considerably higher than other, more reliable, variational calculations.

In an earlier letter [7] we have suggested an alternative method of L.O.C.V. which we have found to give excellent results when applied to the system of Boltzmann particles described above. The purpose of this paper is to describe this method in detail, to show that it is justified on purely variational grounds, and to compare it with the method of Pandharipande. We also apply the method to the more realistic fermion problem of liquid ${ }^{3} \mathrm{He}$ and obtain values for the binding energy and saturation density differing somewhat from other microscopic calculations, but close to the experimental values. We also consider "realistic" neutron matter where we obtain values for the energy per particle considerably lower than those found using the L.O.C.V. of Pandharipande. In the final section of the paper we discuss the reliability and significance of this work and indicate that further tests are at present under way, particularly with regard to a more detailed treatment of the problems of Fermi statistics. For convenience, in the following sections we develop the method for Fermi statistics and describe the simplifications for Boltzmann particles where appropriate.

\section{Variational Method}

Let us consider an infinitely extended system of spin- $\frac{1}{2}$ fermions with no other internal quantum numbers (e.g., neutrons or ${ }^{3} \mathrm{He}$ atoms), interacting through a two-body potential. The system is described by a Hamiltonian, $H$ given by

$$
H=-\frac{\hbar^{2}}{2 m} \sum_{i=1}^{N} \nabla_{i}^{2}+\sum_{i<j=1}^{N} V(i j), \quad N \rightarrow \infty .
$$

The Jastrow correlation function method $[1,2]$ consists of forming a trial, many-body ground-state wavefunction,

$$
\psi=A\left\{\prod_{i<j} f(i j) \prod_{i} \phi_{i}\left(\mathbf{r}_{i}\right)\right\},
$$


where $f$ is a two-body correlation function, the functions $\phi_{i}$ are a complete set of single particle states, and $A$ is the antisymmetrizing operator. The variational principle then gives the ground state energy,

$$
E=\min \frac{\left(\psi^{*}, H \psi\right)}{\left(\psi^{*}, \psi\right)}
$$

Following normal Fermi liquid theory, we consider $N$ fermions normalized in a volume $\Omega$. The uncorrelated single particle states are then

$$
\phi_{i}\left(\mathbf{r}_{i}\right)=\Omega^{-\mathbf{1} / 2} \exp \left(i \mathbf{k}_{i} \cdot \mathbf{r}_{i}\right) \chi_{i}(i),
$$

where $k_{i} \leqslant k_{\mathrm{F}}$, the Fermi momentum, and $\chi_{i}$ is the spin state. The density is given in terms of $k_{F}$ by,

$$
\rho=k_{\mathrm{F}}^{3} / 3 \pi^{2} \text {. }
$$

The energy $E$ is expanded in a cluster series,

where

$$
E=E_{1}+E_{2}+\cdots,
$$

$$
E_{1}=\sum_{i}\left(\phi_{i}^{*},-\frac{\hbar^{2}}{2 m} \nabla^{2} \phi_{i}\right)=\frac{3}{5} \frac{\hbar^{2} k_{\mathrm{F}}^{2}}{2 m} N
$$

and

$$
\begin{aligned}
E_{2}= & \sum_{i<j} C_{2}(i j), \\
C_{2}(i j)= & \left(A \phi_{i}{ }^{*} \phi_{j}{ }^{*}, H_{2}(12) A \phi_{i} \phi_{j}\right), \\
H_{2}= & f^{2}(12)\left\{-\frac{\hbar^{2}}{m}\left[\frac{\nabla_{1}{ }^{2} f(12)}{f(12)}+\frac{\nabla_{1} f(12)}{f(12)} \cdot \nabla_{1}+\frac{\nabla_{2} f(12)}{f(12)} \cdot \nabla_{2}\right]\right. \\
& \left.+\frac{V(12)}{f(12)} f(12)\right\} .
\end{aligned}
$$

We have given the cluster series up to two-body terms [8], and it is our purpose to consider forms for the function $f$ for which truncation at this point is valid.

For potentials of the form

$$
V=\sum_{l} V^{l}(r) P^{l},
$$

where $P^{l}$ is the projection operator for the $l$ th relative partial wave, we must consider a function $f$ of the form

$$
f(\mathbf{r})=\sum_{l} f_{l}(r) P^{l}
$$


In principle, $f$ may also depend on the relative momentum, $k$, of the interacting pair,

$$
k=\frac{1}{2}\left|\mathbf{k}_{i}-\mathbf{k}_{j}\right|,
$$

but such a dependence is unlikely to have a large effect on the two-body energy [9], so we will use the approximation that, for a particular density, $f$ depends only on the relative separation and the relative angular momentum of the interacting pair. Since the $V^{l}(r)$ considered here have at most three different forms, namely, for $l$ is zero, $l$ is odd, and $l$ is even and nonzero, the $l$ dependence of $f(r)$ is similarly restricted to three corresponding forms, although a generalization to include different forms for higher $l$ states is clearly straightforward.

Separating (8) into relative and center-of-mass coordinates, using the Rayleigh partial wave expansion for $\exp (i \mathbf{k} \cdot \mathbf{r})$, and integrating over the center-of-mass and relative angular coordinate gives,

$$
\begin{aligned}
C_{2}(i j)= & \sum_{l}(2 l+1)\left[1-(-1)^{l} \delta_{\alpha \beta}\right] C_{2}{ }^{l}(i j), \\
C_{2}{ }^{l}(i j)= & \frac{4 \pi}{\Omega k^{2}} \int_{0}^{\infty}\left\{-\frac{\hbar^{2}}{m}\left[f_{l}(r) f_{l}^{\prime \prime}(r) p_{l}{ }^{2}(k r)+2 f_{l}(r) f_{l}^{\prime}(r) p_{l}(k r) p_{l}^{\prime}(k r)\right]\right. \\
& \left.+V^{l}(r) f_{l}^{2}(r) p_{l}{ }^{2}(k r)\right\} d r
\end{aligned}
$$

where $p_{l}(x) \equiv x j_{l}(x), j_{l}$ is the spherical Bessel function of order $l$, the primes denote differentiation with respect to $r$, and $\alpha$ and $\beta$ are the spin indices of particles $i, j$.

In order for the method to make physical sense, the functions $f_{l}(r)$ must satisfy the following natural conditions.

(i) $f_{l}(0)$ must be finite, i.e., regular at the origin,

(ii) $f_{l}(r) \underset{r \rightarrow \infty}{\longrightarrow} 1$, and

(iii) $f_{l}(r)$ and $f_{l}^{\prime}(r)$ must be continuous everywhere.

Condition (iii) permits integration by parts on Eq. (12), giving

$$
C_{2}{ }^{l}(i j)=\frac{4 \pi}{\Omega k^{2}} \int_{0}^{\infty}\left\{\frac{\hbar^{2}}{m} f_{l}^{\prime 2}(r)+V^{l}(r) f_{l}^{2}(r)\right\} p_{l}{ }^{2}(k r) d r,
$$

where the integrated term is zero due to conditions (i) and (ii) above.

From (8), summing over the four possible spin states for a pair of spin- $\frac{1}{2}$ fermions and summing over the odd and nonzero even values of $l$, we have

$$
E_{2}=\sum_{\mathbf{k}_{i} \neq \mathbf{k}_{j}} \frac{1}{\Omega} \int_{0}^{\infty} \sum_{t=0}^{2}\left\{\frac{\hbar^{2}}{m} f_{t}^{\prime 2}(r)+V^{t}(r) f_{t}^{2}(r)\right\} b_{t}(\mathbf{k}, \mathbf{r}) d \mathbf{r},
$$


where $t=0,1,2$ corresponds, respectively, to $l$ being zero, odd, and even (but nonzero). The functions $b_{t}(\mathbf{k}, \mathbf{r})$ are given by,

$$
\begin{aligned}
& b_{0}(\mathbf{k}, \mathbf{r})=j_{0}^{2}(k r), \\
& b_{1}(\mathbf{k}, \mathbf{r})=3 \sin ^{2}(\mathbf{k} \cdot \mathbf{r}), \\
& b_{2}(\mathbf{k}, \mathbf{r})=\cos ^{2}(\mathbf{k} \cdot \mathbf{r})-j_{0}^{2}(k r) .
\end{aligned}
$$

In the continuum limit,

$$
\sum_{\mathbf{k}_{i}} \rightarrow\left(\Omega /(2 \pi)^{3}\right) \int_{0}^{k_{F}} d \mathbf{k}_{i}
$$

and so performing the integrations over $\mathbf{k}_{i}$ and $\mathbf{k}_{j}$ implied in Eq. (14) gives,

$$
E_{2} / N=\left(\rho \pi / 2{k_{\mathrm{F}}}^{2}\right) \int_{0}^{\infty} \sum_{t=0}^{2}\left\{\left(\hbar^{2} / m\right) f_{t}^{\prime 2}(r)+V^{t}(r) f_{t}^{2}(r)\right\} a_{t}^{2}\left(k_{\mathrm{F}} r\right) d r
$$

where

$$
\begin{aligned}
& a_{0}(x)=3\left\{1-\frac{1}{x^{2}}-\frac{\sin ^{2} x}{x^{4}}+\frac{2 \sin x \cos x}{x^{3}}\right\}^{1 / 2} \\
& a_{1}(x)=\left\{3\left[x^{2}-\frac{9(\sin x-x \cos x)^{2}}{x^{4}}\right]\right\}^{1 / 2} \\
& a_{2}(x)=\left\{x^{2}+9\left[\frac{(\sin x-x \cos x)^{2}}{x^{4}}-1+\frac{1}{x^{2}}+\frac{\sin ^{2} x}{x^{4}}-\frac{2 \sin x \cos x}{x^{3}}\right]\right\}^{1 / 2}
\end{aligned}
$$

Varying the functions $f_{t}(r)$ to minimize $E_{2} / N$ gives the three uncoupled EulerLagrange equations,

$$
\begin{array}{r}
-\left(\hbar^{2} / m\right)\left\{f_{t}^{\prime \prime}(r) a_{t}\left(k_{\mathrm{F}} r\right)+2 a_{t}^{\prime}\left(k_{\mathrm{F}} r\right) f_{t}^{\prime}(r)\right\}+V^{t}(r) a_{t}\left(k_{\mathrm{F}} r\right) f_{t}(r)=0, \\
t=0,1,2,
\end{array}
$$

or, equivalently,

$$
\frac{d^{2}}{d r^{2}} g_{t}(r)-\left\{\frac{a_{t}^{\prime \prime}\left(k_{\mathrm{F}} r\right)}{a_{t}\left(k_{\mathrm{F}} r\right)}+\frac{m}{\hbar^{2}} V^{t}(r)\right\} g_{t}(r)=0, \quad t=0,1,2,
$$

where $g_{t}(r)=a_{t}\left(k_{\mathrm{F}} r\right) f_{t}(r)$, and the primes denote differentiation with respect to $r$.

It is well known that if Eq. (20) is solved for a potential with an attractive tail then there are no solutions with the correct asymptotic behavior of $f_{t}(r \rightarrow \infty) \rightarrow 1$. This problem, the "Emery Difficulty" [3], results from the fact that the cluster expansion for the energy has been truncated after two-body terms and the EulerLagrange equation gives an $f_{t}(r)$ for which this truncation is not justified. 


\section{Constrained Variation}

In dense matter, where the interparticle potentials have a strongly repulsive core, it is this core which is responsible for most of the short-range correlations and so, with the truncation of the cluster expansion after two-body terms, it is unrealistic to allow the attractive part of the potential to have a large effect on the form of the correlation function. Thus we impose the constraint on $f_{l}(r)$ that

$$
0 \leqslant f_{t}(r) \leqslant 1
$$

This constraint has been widely used in nuclear matter and other many-body calculations $[2,8,10]$ where a particular form has often been assumed for the function $f$ [e.g., $f(r)=1-e^{-a(r-c)} ; r>c$ ], and it is found to give reasonable results in the density range of equilibrium nuclear matter.

Constraint (21) is sometimes objected to on the grounds that the exact expression for the energy of the system, written in terms of the exact pair distribution function $g(\mathbf{r})$, reduces to our expression (14) in the approximation $g_{t}(\mathbf{r}) \rightarrow f_{t}^{2}(r)$. It is then argued, in the familiar case of liquid helium for example, that the peak which develops in $g(\mathbf{r})$ should be reflected in our "bare" correlation function $f(\mathbf{r})$. As long as we are interested only in a calculation of the energy of the system, however, this argument is spurious for the following reasons. Peaks in pair distribution functions can be thought of as arising primarily from two related sources, namely long-range effects and the requirement of overall normalization of the exact wavefunction. Our method is clearly designed to account for short-range correlations and effects coming from long-range forces are clearly excluded from consideration. On the other hand, the peak which $g(\mathbf{r})$ develops, in liquid helium, for example, is probably mostly due to the overall normalization requirement and not to the attractive part of the potential. It is likely that $g(\mathbf{r})$ would have a large peak even in the complete absence of the attractive well in this case. In this respect it is certainly true that with the imposition of constraint (21) it is impossible to impose exact normalization of the wavefunction within the two-body approximation. It is probable, however, that this lack of normalization has a small effect on the computation of the energy for nucleons at around nuclear matter density [10], although for liquid ${ }^{3} \mathrm{He}$ this lack of normalization may be a more serious deficiency. It is true that, were we interested in a realistic calculation of $g_{t}(r)$, the expression $f_{t}^{2}(r)$ would be a bad approximation, but it could still be used as the input, for example, to a higher selfconsistent approximation for $g(\mathbf{r})$.

It is worth noting that the L.O.C.V. method of Pandharipande which is described in Section 5, and in comparison with which we are chiefly interested at this stage, does not explicitly impose constraint (21). However, in the calculations described in Section 6 we have compared results by our method with the L.O.C.V. prescrip- 
tion of Pandharipande. We find that for all the systems studied the correlation functions obtained in the L.O.C.V. prescription in fact always obey condition (21) except at very low densities, where our method is not designed to apply.

Clearly, any constraint imposed at the two-body level becomes irrelevant if all of the omitted terms in the cluster expansion are also evaluated. Our motivation in imposing constraint (21) is to keep these higher-order terms small. Compared with an unconstrained calculation we hope to be able to include in our effective two-body energy the dominant contributions arising from these terms. Within this context it is interesting to compare our imposition of constraint (21) with recent calculations of Clark, Lam, and ter Louw [10]. These authors have performed variational calculations on model nuclear matter systems using particular parametrized forms of their correlation functions $f(r)$ and minimizing with respect to these parameters. In particular, they have compared results with forms of the correlation functions $f(r)$ which do and do not contain peaks, i.e., which do and do not satisfy Eq. (21). These authors find, by doing perturbation theory within their correlation function basis (and working to second-order perturbation theory evaluated in the two-body cluster approximation), that so long as the leading term in the perturbation series, namely, the Jastrow energy expectation value, is evaluated through the three-body cluster order, their results are essentially independent of whether their correlation functions contain a peak. or not. In so far as our contraints are designed to include the dominant pieces of the higher clusters (which are themselves constrained to be small), these results indicate that imposition of constraint (21) is certainly not very severe for an evaluation of the energy.

While constraint (21) is sufficient to overcome the "Emery Difficulty," it may not be, of itself, sufficient to ensure that the truncation of the cluster expansion in Eq. (6) is justified, particularly at densities where, in an uncorrelated system, there is a high probability of particles entering the range of one another's repulsive cores. Under such conditions, the dominant effect of the introduction of two-body correlations should be to expel any particle which is within the range of the repulsive core of another. Thus, if we restrict the average number of particles expelled by the correlations from the core radius $R_{\mathrm{c}}$ of any particle to be less than 1 , then, on the average, we will be restricting correlations to be between nearest neighbors, and truncation of the cluster expansion should hopefully be justified. Calculated in the two-body approximation this gives,

$$
\left(\pi \rho / k_{\mathrm{F}}^{2}\right) \sum_{t=0}^{2} \int_{0}^{R_{\mathrm{c}}}\left(1-f_{t}^{2}(r)\right) a_{t}^{2}\left(k_{\mathrm{F}} r\right) d r \leqslant 1,
$$

where the functions $a_{t}$ are given by Eqs. (18), and $R_{\mathrm{c}}$ is the radius of the repulsive core of the potential.

This constraint has been derived on average physical grounds and so is presum- 
ably more directly applicable to Boltzmann particles (in which case $k_{\mathrm{F}}^{-2} a_{t}^{2}\left(k_{\mathrm{F}} r\right) \rightarrow$ $4 r^{2}$, and the sum over $t$ disappears), where the additional complication of spinstatistics does not arise. For this reason we first apply constraint (22) to the Boltzmann homework problem and its subsequent success suggests that a direct application to Fermi problems is worthwhile.

Since this constraint, together with the constraint $f_{t}(r) \leqslant 1$, forces $f_{t}(r)$ to tend rapidly to unity, we may replace the upper limit of the integral by $+\infty$ with the effect of making the constraint only very slightly stronger. Constraint (22) introduces the same Lagrange multiplier into each of Eqs. (20) so that we must solve

$$
\frac{d^{2}}{d r^{2}} g_{t}(r)-\left\{\frac{a_{t}^{u}\left(k_{\mathrm{F}} r\right)}{a_{t}\left(k_{\mathrm{F}} r\right)}+\frac{m}{\hbar^{2}}\left[V^{t}(r)+\lambda\right\}_{\}} g_{t}(r)=0, \quad t=0,1,2,\right.
$$

subject to the constraints

$$
\begin{gathered}
0 \leqslant f_{t}(r) \leqslant 1 \\
\frac{\pi \rho}{k_{\mathrm{F}}{ }^{2}} \int_{0}^{\infty} \sum_{t=0}^{2}\left(1-f_{t}^{2}(r)\right) a_{t}{ }^{2}\left(k_{\mathrm{F}} r\right) d r \leqslant 1 .
\end{gathered}
$$

Equations (23)-(25) form the basis of our constrained variational method, and they are now amenable to exact functional solution, as we describe in the next section.

\section{Method of SOLution}

Equation (23) has two linearly independent solutions, but one of these is singular at the origin and so is excluded by condition (i) of Section 2 , in order to ensure continuity at $r=0$. Thus

$$
f_{t}(r)=A_{t} \frac{g_{t}(r)}{a_{t}\left(k_{\mathrm{F}} r\right)},
$$

where $g_{t}(r)$ is the solution of (23) which is regular at the origin, and $A_{t}$ is a constant. Since we are considering potentials with a strongly repulsive core, we introduce constraint (24) by supposing a distance $d^{t}$ such that for $r>d^{t}$ minimization of the two-body energy with $f_{t}(r) \leqslant 1$ is obtained with $f_{t}(r) \equiv 1$. That such a $d^{t}$ exists is evident for a potential with an attractive tail, since constraint (25) ensures that $f_{t}\left(a^{t}\right)=1$ for some $a^{t}$ inside the attractive tail. Then for $r>a^{t}$ minimization is obtained with $f_{t} \equiv 1$, since distorting $f_{t}(r)$ away from unity such that $f_{t}(r)<1$ increases both terms in the energy expression (17). Notice that the condition that $f_{t}^{\prime}(r)$ be continuous at $r=a^{t}$ does not affect this argument (see the Appendix). Thus $d^{t} \leqslant a^{t}$. 
A similar argument holds for a potential which is purely repulsive, provided that the repulsion falls off sufficiently rapidly as $r \rightarrow \infty$, but in this case distorting $f_{t}(r)$ away from unity for large $r$ must be compensated for by an increase in $f_{t}(r)$ for smaller $r$ (because of constraint (25)) and this will clearly be energetically unfavorable for a rapidly decreasing, repulsive potential.

The constant $A_{t}$ is chosen such that $f_{t}\left(d^{t}\right)=1$, and $d^{t}$ is made as large as possible with $f_{t}\left(r<d^{t}\right) \leqslant 1$. Now if $f_{t}^{\prime}\left(r==d^{t}\right)<0$, then $f_{t}\left(d^{t}-h\right)>f_{t}\left(d^{t}\right)=1, h>0$, which contradicts $f_{t}(r) \leqslant 1$, and if $f_{t}^{\prime}\left(r=d^{t}\right)>0$, then $d^{t}$ may be increased to $d^{t}+h$ when $f_{t}\left(d^{t}-h\right)<f_{t}\left(d^{t}\right)=1$, so that $d^{t}$ was not as large as possible. Thus $d^{t}$ is the point where,

$$
f_{t}^{\prime}\left(d^{t}\right)=0
$$

Now from Eq. (23),

$$
f_{t}^{\prime}(r)=A_{t}\left\{\frac{g_{t}^{\prime}(r)}{a_{t}\left(k_{\mathrm{F}} r\right)}-\frac{a_{t}^{\prime}\left(k_{\mathrm{F}} r\right)}{a_{t}{ }^{2}\left(k_{\mathrm{F}} r\right)} g_{t}(r)\right\} .
$$

Our method is to solve (23) for $g_{t}(r)$ and determine $d^{t}$ so that,

$$
g_{t}^{\prime}\left(d^{t}\right)=\frac{a_{t}^{\prime}\left(k_{\mathrm{F}} d^{t}\right)}{a_{t}\left(k_{\mathrm{F}} d^{t}\right)} g_{t}\left(d^{t}\right)
$$

We then determine $A_{t}$ so that $f_{t}\left(d^{t}\right)=1$, and set $f\left(r>d^{t}\right) \equiv 1 . \lambda$ is initially set to 0 , and if constraint (25) is not satisfied, $\lambda$ is varied iteratively, solving for $f_{t}(r)$, $A_{t}$, and $d^{t}$ at each step, until

$$
\sum_{t=0}^{2}\left(\rho \pi / k_{\mathrm{F}}{ }^{2}\right) \int_{0}^{d^{t}}\left(1-f_{t}^{2}(r)\right) a_{t}^{2}\left(k_{\mathrm{F}} r\right) d r=1,
$$

where the upper limit of the integral becomes $d^{t}$ because $f_{t}\left(r>d^{t}\right) \equiv 1$.

\section{Comparison with L.O.C.V. of Pandharipande}

Pandharipande $[4,11]$ solves an equation similar to Eq. (23) with the constraint $f_{t}(r>d) \equiv 1$ for some $d$, but he determines $d$ from the condition that on the average there should be exactly one particle inside the correlation volume defined by $d$. In the two-body approximation this gives

$$
\left(\rho \pi / k_{\mathrm{F}}^{2}\right) \int_{0}^{d} \sum_{t=0}^{2} f_{t}^{2}(r) a_{t}^{2}\left(k_{\mathrm{F}} r\right) d r=1,
$$

and he introduces a parameter $\lambda^{t}$, similar to the $\lambda$ above, but uses this to fix $f_{t}^{\prime}(d)=0$. 
Constraints (30) and (31) are clearly very similar and, insofar as the $t$ dependence of our $d$ may be ignored, are identical when

$$
\left(\rho \pi / k_{\mathrm{F}}^{2}\right) \int_{0}^{d} \sum_{t=0}^{2} a_{t}^{2}\left(k_{\mathrm{F}} r\right) d r=2 .
$$

It is interesting to note that this occurs when $d \sim 1.2 \times r_{0}$, where $r_{0}=(4 \pi \rho / 3)^{-1 / 3}$ is the unit radius, and that this is of the same order as the value for $d$ which Pandharipande commonly finds in his calculations. Condition (31), however, has the peculiar feature that if we increase the depth of the correlations which we use, then, in order, on the average, to restrict correlations to the nearest neighbor, we must increase the range of the correlations. It is unlikely that these two effects would produce counteracting effects on the higher-order terms in the cluster expansion, particularly in the high density limit.

Pandharipande's method involves minimizing the two-body energy as given in Eq. (12) for $r$ between 0 and $d$ with the boundary conditions: $f_{l}(0)=$ finite, $f_{l}(d)=1$, and $f_{l}^{\prime}(d)=0$. This is an over-prescribed problem (since the corresponding Euler-Lagrange equation is second order and there are three boundary conditions), and, in general, no $f_{l}(r)$ exists which exactly minimizes Eq. (12) with these boundary conditions. Pandharipande circumvents this difficulty by adding a parameter, $\lambda^{l}$, to the two-body potential, which he varies to satisfy the condition $f_{l}^{\prime}(d)=0$, and he justifies this procedure by analogy with perturbation theory. Such a procedure is not justified on variational grounds, however, for the following reason. We may obtain a lower bound for $C_{2}{ }^{l}(i j)$ by considering Eq. (13) and removing the boundary condition that $f_{l}^{\prime}(d)=0$. It is true that, without this condition, (12) and (13) are not equivalent (since then $f_{l}^{\prime}(r)$ is not continuous), but we are trying to find a lower bound for $C_{2}{ }^{l}(i j)$ with the condition imposed, and so we may work with either (12) or (13). Removing the condition in (12) is not useful because we simply obtain a minimum of minus infinity. Minimizing (13) without the boundary condition $f_{l}^{\prime}(d)=0$ is equivalent to solving an equation similar to (23) but without the parameter $\lambda$. The Appendix shows that we can form an $f_{l}(r)$ satisfying $f_{l}^{\prime}(d)=0$ which gives a $C_{2}{ }^{l}(i j)$ which is vanishingly different from this lower bound and so this lower bound is the greatest lower bound and should be taken as the minimum. Thus the introduction of $\lambda$ into Pandharipande's method is not necessary and is therefore not justified variationally.

In the variational method described here, however, the difficulty of the nonexistence of an exact minimum does not arise. We prove that the subsidiary constraints (24) and (25) imply that $d^{t}$ must be chosen to satisfy $f_{t}^{\prime}\left(d^{t}\right)=0$, and so we are left to solve a second-order differential equation with just two boundary conditions. It appears that the introduction of the parameter $\lambda$ is necessary in Pandharipande's method in order to counteract, in a rather arbitrary way, the behavior of condition 
(31). In contrast, by reversing the roles played by $\lambda$ and $d$, we have been able to justify the inclusion of both on variational grounds alone.

\section{RESULTS}

In an attempt to comparc different variational methods, scveral groups have recently applied their variational methods to a hypothetical system of neutrons treated as Boltzmann particles and interacting through the repulsive part of the Reid [12] soft-core ${ }^{1} S_{0}$ potential. The results of Chakravarty, Miller, and Woo (CMW) [13], Shen and Woo (SW), and Pandharipande (P) have been reported and compared in a recent paper by Shen and Woo [6]. These groups all use different variational methods and all except $P$ involve the explicit calculation of many-body terms in the cluster series.

The constrained variational method which we have described in the previous sections for fermions applies directly to Boltzmann particles if we remove the summation over $t$ from all of the equations and make the substituton $k_{\mathrm{F}}^{-2} a_{t}^{2}\left(k_{\mathrm{F}} r\right) \rightarrow$ $4 r^{2}$. In fact, as we have already remarked, constraint (22), because it is justified on average physical grounds, should be more directly applicable to Boltzmann particles.

In Fig. 1 we compare the results obtained using the method presented above with those of CMW, SW, and P, as taken from [6, Fig. 1]. As in [6], SW is taken as standard and the deviation of the other results from theirs is plotted. Figure 2 shows the absolute energics obtaincd using the present method and using P's

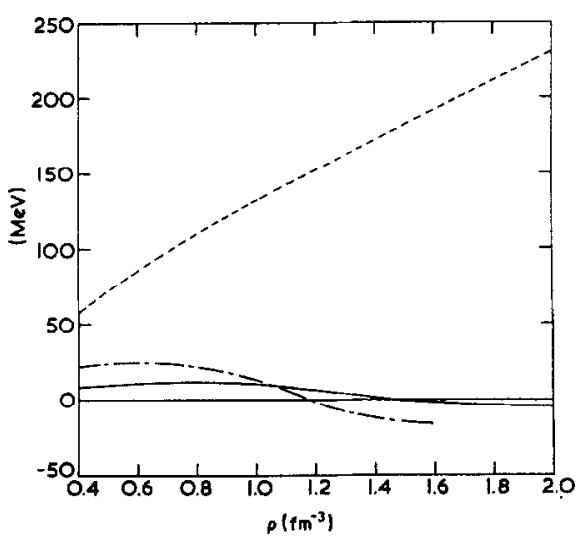

FIG. 1. Comparison of the results of several groups for the hypothetical gas of neutrons obeying Boltzmann statistics (taken from [6, Fig. 1]): - - $\left.E / N)_{\text {present }}-E / N\right)_{\text {sw }} ;------$, $\left.\left.\left.E / N)_{\mathrm{P}}-E / N\right)_{\mathrm{sw}} ;-\cdots \cdot \cdots \cdot-\cdot E / N\right)_{\mathrm{CMW}}-E / N\right)_{\mathrm{sw}}$. 


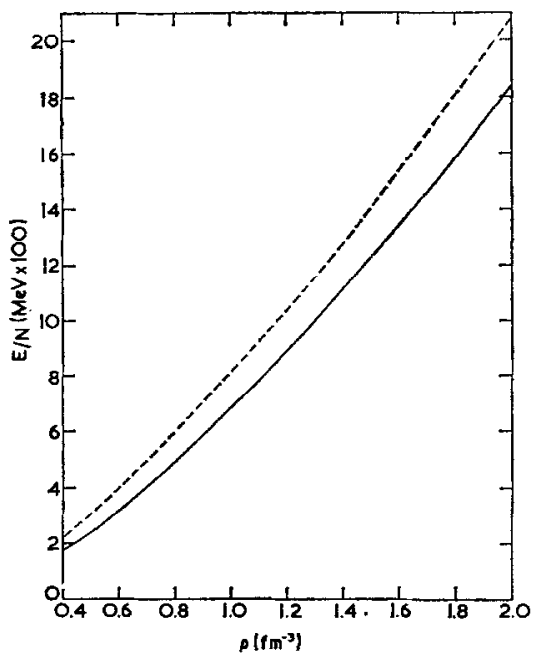

Fig. 2. Total energy per particle for the hypothetical gas of neutrons obeying Boltzmann statistics: —_- present method; - - - - -, Pandharipande's method.

method. The results of SW and CMW are not distinguishable from ours on this scale. It is clear that our results are in excellent agreement with those of CMW and $\mathrm{SW}$, but differ appreciably from those of $\mathrm{P}$. Whilst this is admittedly an extremely simple model with none of the complexities of spin and angular momentum dependence present in "realistic" neutron matter, the agreement with CMW and SW is an extremely encouraging indication that we have indeed succeeded in restricting the effect of higher-order terms in the cluster series to such an extent that a similar calculation on realistic fermion systems might be expected to give credible results.

Figure 3 and Table I give the results obtained for a calculation of the binding energy of liquid ${ }^{3} \mathrm{He}$ using the Lennard-Jones potential [14],

$$
V(r)=C\left\{(\sigma / r)^{12}-(\sigma / r)^{6}\right\},
$$

where $C=40.88^{\circ} \mathrm{K}, \sigma=2.556 \AA$.

Using just two correlation functions, one each for the singlet and triplet states, we obtain a minimum for the energy per particle, $(E / N)_{\min }$, of $-3.18^{\circ} \mathrm{K}$ at a Fermi momentum of $0.81 \AA^{-1}$, corresponding to a density of 0.018 atoms $\AA^{-3}$. In Fig. 3 we also show the results of a similar calculation using the method of L.O.C.V. of Pandharipande, but we have avoided the unnecessary approximation of defining an effective two-body interaction which is independent of $k$ as is done in [4]; that is, we have solved [4, Eq. (10)] subject to [4, conditions (7) and (12)] and used the functions $f_{l}(r)$ so obtained to calculate the two-body energy as given by 
TABLE I

Liquid ${ }^{3} \mathrm{He}$ using the Lennard-Jones Potential

\begin{tabular}{ccc}
\hline$k_{F}\left(\AA^{-1}\right)$ & $\lambda\left({ }^{\circ} \mathrm{K}\right)$ & $E / N\left({ }^{\circ} \mathrm{K}\right)$ \\
\hline 0.70 & 0.0 & -1.96 \\
0.72 & 0.0 & -2.23 \\
0.74 & 0.0 & -2.51 \\
0.76 & -1.00 & -2.78 \\
0.78 & -2.45 & 3.03 \\
0.80 & -5.25 & -3.17 \\
0.82 & -9.65 & -3.16 \\
0.84 & -16.03 & -2.90 \\
0.86 & -24.47 & -2.35 \\
0.88 & -35.53 & -1.39 \\
0.90 & -52.67 & 0.39 \\
\hline
\end{tabular}

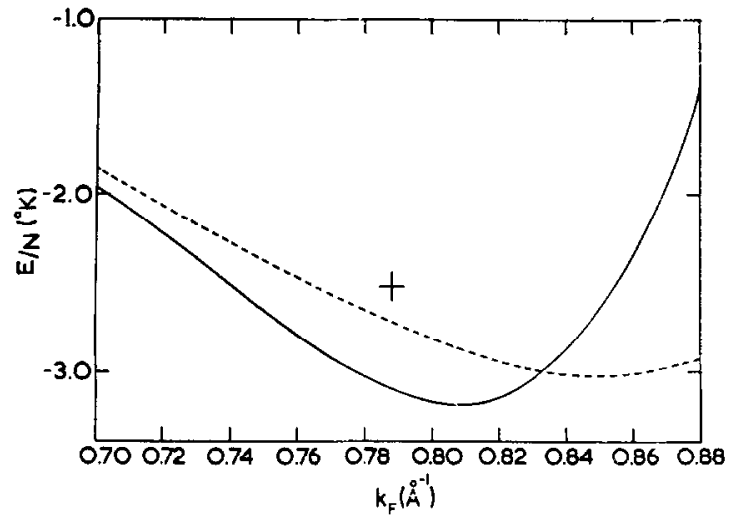

FIG. 3. Liquid ${ }^{3} \mathrm{He}$ using Lennard-Jones potential: ___, present method; _- ...-, Pandharipande's method; + , experimental point.

Eq. (17) above. This explains the small discrepancy between the results given here, and those given in [4].

Our results are clearly fairly close to the experimental values for the binding energy of $-2.52^{\circ} \mathrm{K}$ and saturation density of $0.0164 \AA^{-3}$. However, the results of more advanced microscopic calculations using the Lennard-Jones potential give less binding at a lower saturation density. For example, using a molecular dynamics method and a perturbation correction for Fermi statistics, Schiff and Verlet [15] 
find a binding energy of $-1.35^{\circ} \mathrm{K}$ at a density of $0.014 \AA^{-3}$. Since in suitably scaled variables the Lennard-Jones potential is both strong and very long-ranged, one should probably not expect any low order approximation to give accurate results, and we suggest that the fact that we obtain binding at all at a reasonable density is encouraging. We also note that the very high density behavior of our energy is probably more sensible than that of Pandharipande, indicating again that our constraints are more appropriate.

TABLE II

Neutron Matter Using the Modified Reid Potential

\begin{tabular}{ccc}
\hline$k_{F}\left(\mathrm{~F}^{-1}\right)$ & $\lambda(\mathrm{MeV})$ & $E / N(\mathrm{MeV})$ \\
\hline 1.0 & 0.0 & 7.15 \\
1.5 & 0.0 & 14.7 \\
2.0 & 0.0 & 27.5 \\
2.5 & -5.51 & 50.1 \\
3.0 & -49.7 & 99.8 \\
3.5 & -155 & 205 \\
4.0 & -337 & 405 \\
4.5 & -587 & 735 \\
5.0 & -907 & 1231 \\
\hline
\end{tabular}

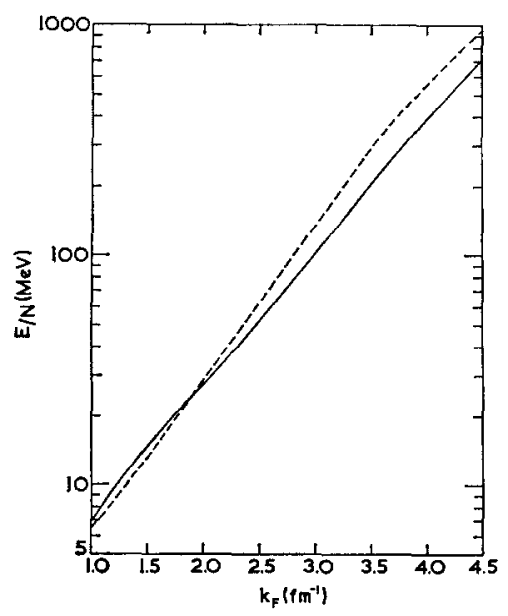

FIG. 4. Neutron matter using modified Reid potential: Pandharipande's method. present method; -------, 
Table II and Fig. 4 give the results of the calculation of the energy per particle of pure neutron matter, treating the neutrons correctly as fermions, and using a modified version of the Reid soft-core, isospin triplet potential [12] as suggested by Pandharipande [9]. This potential has the Reid ${ }^{1} S_{0}$ for $l=0$ states, the Reid ${ }^{1} D_{2}$ for nonzero, even- $l$ states, and the central part of the Reid ${ }^{3} P_{2}-{ }^{3} F_{2}$ for the odd- $l$ states. The results of a similar calculation using the method of Pandharipande as described in [4] are also plotted in Fig. 4 and it is clear that at higher densities our results again lie considerably below those of Pandharipande. In Fig. 5 we show a plot of the $l=0$ correlation functions, $f_{0}(r)$, as obtained using the present method and using the method of Pandharipande, at a Fermi momentum of $k_{\mathrm{F}}=2.5 \mathrm{~F}^{-1}$. We find, generally, for all but the lowest densities $\left(k_{\mathrm{F}}<1.5 \mathrm{~F}^{-1}\right)$, that the $f_{t}(r)$ obtained using Pandharipande's method do, in fact, satisfy the condition imposed in our method, that $f_{t}(r) \leqslant 1$.

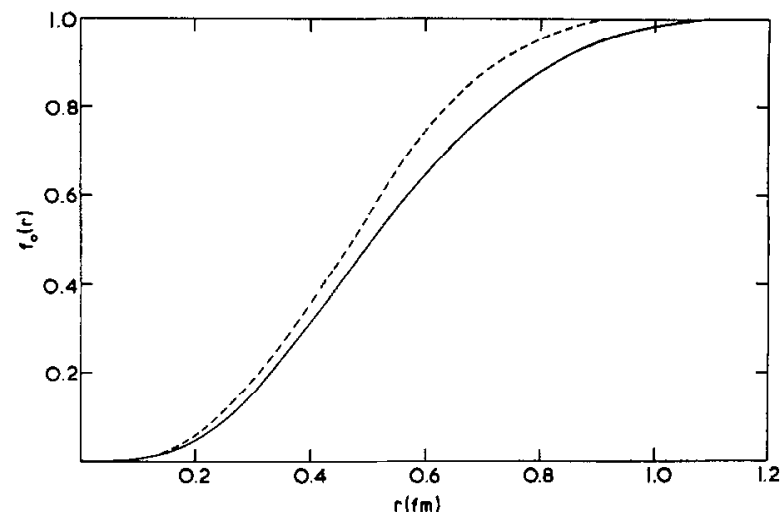

Fig. 5. The $l=0$ correlation functions for the calculation of Fig. 4 at $k_{\mathrm{P}}=2.5 \mathrm{~F}^{-1}$ : present method; - - - --, Pandharipande's method.

\section{Discussion}

The major uncertainty in the formalism described above is clearly the total absence of any explicit calculation of the higher-order terms in the cluster series. If we accept the idea that the final sum over all the terms in the cluster series, were we able to perform such a summation, would not depend very critically upon the exact form of the two-body correlation function, then we can adopt the following viewpoint. We regard the introduction of our constraints on the correlation function as restricting the magnitude of the sum of the higher-order terms in the cluster series in some self-consistent way; and we suggest that the introduction of 
the constraints effectively subsumes part of the higher-order contribution, which would occur if we used the physically correct two-body correlation function, into the two-body energy obtained with our constraint. This philosophy bears obvious analogy to that adopted in the "renormalized" version of many-body perturbation theory, typified by the Brueckner-Bethe-Goldstone [16] theory, which has proved so useful in nuclear matter calculations. In both these theories, however, it is very difficult to check at the end of the calculation whether one has, in fact, obtained the desired close approximation to the final sum of the energy series, since the introduction of either physically motivated constraints in the present case, or single particle potentials in the case of Brueckner theory, will destroy the original ordering scheme for the energy series, and may imply cancellations between terms which are individually large.

Having said this, it is, on the other hand, quite clearly unsatisfactory to impose constraints, which by their nature are intuitively inspired, and then to trust to luck that the cluster expansion can be terminated at the two-body level. The only real test of any constrained variational calculation of the sort described here is direct comparison with an exact unconstrainted calculation for the same system. For the "homework" problem of neutrons interacting as Boltzmann particles described above, the results of Shen and Woo [6] can probably be regarded as "exact," and it is extremely encouraging that in this case our results, by comparison, are very good indeed, and clearly superior to those of Pandharipande.

The situation with regard to liquid ${ }^{3} \mathrm{He}$ is by no means so transparent. It is probable that an unconstrained cluster expansion for this system would be very slowly convergent and it is therefore not surprising that our results differ from other higher-order calculations with the Lennard-Jones potential. It is presumably coincidental that our results lie so close to the experimental values. The fact that we obtain more binding at a higher equilibrium density than, for example, Schiff and Verlet [15] we take as an indication that constraint (22) when applied to such a dense Fermi system is rather too weak to adequately constrain the higher order terms to be small. We note also that allowing "overshoot," as the exact two-body distribution function would require, would only increase this discrepancy.

For the "realistic" model of neutron matter considered here, again for the density range of interest no exact calculations exist of either the unconstrained variational or the many-body perturbation theory type. ${ }^{1}$ Nuclear matter has, however, been well studied up to densities around the equilibrium value $\left(k_{\mathrm{F}} \leq 1.4 \mathrm{~F}^{-1}\right)$, by the linked-cluster perturbation theory of Brueckner, Bethe, and Goldstone; in this context the familiar convergence parameter $\kappa$, proportional to the volume of the "wound" wavefunction [16], is often defined. It is perhaps worth pointing out that

\footnotetext{
${ }^{1}$ Pandharipande and Bethe [Phys. Rev. C 7 (1973), 1312] have, however, given hypernetted chain results for both this system and liquid ${ }^{3} \mathrm{He}$.
} 
comparable convergence parameters can also be defined within the framework of Jastrow variational theory, although they only have any real meaning for unconstrained calculations, for the reasons already cited. Two popular competing choices of such convergence parameters are

$$
\begin{aligned}
k & \equiv \sum_{i j}\left(i j\left|A\left[1-f^{2}(12)\right] A\right| i j\right) / N \\
& =\sum_{t=0}^{2}\left(\pi \rho / k_{\mathrm{F}}{ }^{2}\right) \int_{0}^{\infty}\left[1-f_{t}^{2}(r)\right] a_{t}{ }^{2}\left(k_{\mathrm{F}} r\right) d r,
\end{aligned}
$$

and

$$
\begin{aligned}
\kappa & \equiv \sum_{i j}\left(i j\left|A[1-f(12)]^{2} A\right| i j\right) / N \\
& =\sum_{t=0}^{2}\left(\pi \rho / k_{\mathrm{F}}^{2}\right) \int_{0}^{\infty}\left[1-f_{t}(r)\right]^{2} a_{t}^{2}\left(k_{\mathrm{F}} r\right) d r .
\end{aligned}
$$

The parameter $\kappa$ is evidently the exact Jastrow analog of the Brueckner-BetheGoldstone smallness parameter. For our constrained calculation, condition (25) that we have imposed shows that the parameter $k$ has been set less than or equal to unity, and for the higher density ranges, $k=1$ has been found necessary. It is clear that constraints (24) and (25) together imply also $\kappa<1$, which perhaps provides another indication of how the constraints that we have imposed act to suppress higher-order terms and thereby improve the convergence of the cluster expansion. While it is certainly naive to treat $\kappa$ as a real convergence parameter in such a constrained calculation, it is probably true that as long as it remains suitably small, it does provide an indication of the rate of convergence.

Bearing this warning in mind, we show in Fig. 6 the parameter $\kappa$ plotted as a function of Fermi momentum for the "realistic" model of neutron matter already described. It is encouraging that even at $k_{\mathrm{F}}=4.0 \mathrm{~F}^{-1}$, the parameter $\kappa$ does not exceed 0.3. Also plotted in Fig. 6 is the analogous quantity obtained using the method of Pandharipande, and it is interesting to note that over most of the density range of interest the $\kappa$ of Pandharipande is approximately one-half of ours. However, because the method of Pandharipande is not strictly a variational method, as already noted, we do not take this result seriously. For all the other reasons already cited we believe the present method superior to that of Pandharipande, and hence the comparative plots of $\kappa$ probably again only indicate the wariness with which one should use the smallness of this parameter as a test of convergence.

In conclusion, we feel that the method of lowest-order constrained variation presented in this work redresses the objections that are open to the method of Pandharipande, and puts the theory on a firm basis, both mathematically and physically. We have suggested an integral constraint which is justified on simple 


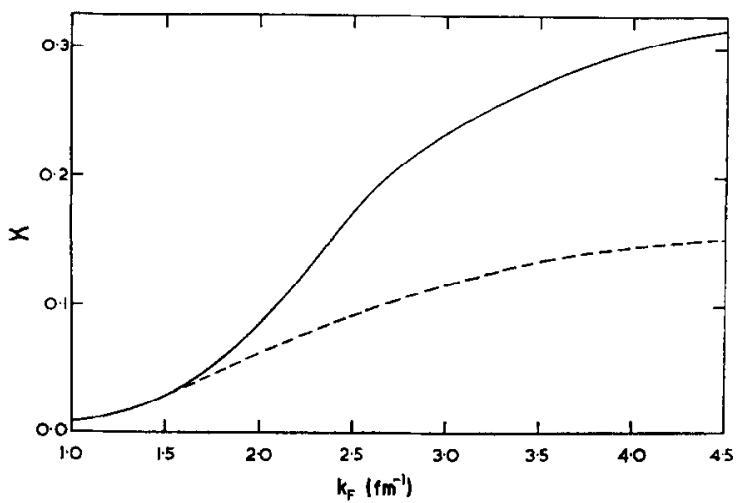

FIG. 6. The convergence parameter $\kappa$ for neutron matter using the modified Reid potential: , present method; ------, Pandharipande's method.

physical grounds and which gives reliable results when applied to the Boltzmann homework problem. We find indications that when applied to such dense Fermi systems as liquid ${ }^{3} \mathrm{He}$ this constraint may be rather too weak and calculations on other model fermion systems which attempt to investigate the most suitable incorporation of Fermi statistics in the present method will appear elsewhere [17].

\section{APPENDIX}

Suppose that solving Eq. (23) from zero to $d$ and then setting $f(r) \equiv 1$ for $r>d$ produces an $f(r)$ as shown in Fig. 7L (i.e., continuous, but with a first derivative discontinuous at $r=d$ ). Then, instead of using this $f(r)$, we use $f_{1}(r)$ as shown in Fig. 7R. $f_{1}(r)$ differs from $f(r)$ only in the small region $\epsilon$ about $r=d$, so that $f_{1}(r)$ is continuous and has a continuous first derivative. Now from Eq. (17),

$$
E_{2}(f) / N=\int_{0}^{\infty} F\left(f^{\prime}, f, r\right) d r
$$
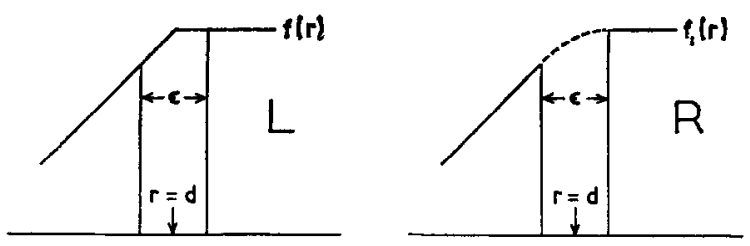

FIG. 7. Method of giving $f(r)$ a continuous first derivative. 
so,

$$
\left|E_{2}(f) / N-E_{2}\left(f_{1}\right) / N\right|=\left|\int_{d-\epsilon / 2}^{d+\epsilon / 2}\left\{F\left(f^{\prime}, f, r\right)-F\left(f_{1}^{\prime}, f_{1}, r\right)\right\} d r\right|
$$

$f, f^{\prime}, f_{1}, f_{1}^{\prime}$ are all clearly bounded independently of $\epsilon$ in the region $\epsilon$ and Eq. (17) shows that both $F\left(f^{\prime}, f, r\right)$ and $F\left(f_{1}^{\prime}, f_{1}, r\right)$ are also bounded in this region. Thus,

$$
\left|E_{2}(f) / N-E_{2}\left(f_{1}\right) / N\right| \leqslant A \epsilon,
$$

for some fixed $A$. Then letting $\epsilon \rightarrow 0$ shows that for any continuous $f(r)$ we may generate an $f_{1}(r)$ with a continuous first derivative which gives an $E_{2} / N$ in Eq. (17) which is vanishingly different from that obtained with $f(r)$, and which differs from $f(r)$ only in a vanishingly small region about $r=d$.

\section{ACKNOWLEDGMENTS}

The authors wish to thank Drs. H. B. Ghassib and M. R. Strayer for several helpful discussions.

\section{REFERENCES}

1. R. JASTROW, Phys. Rev. 98 (1955), 1478.

2. J. W. Clark and P. Westhaus, Phys. Rev. 141 (1966), 833.

3. V. J. EMERY, Nucl. Phys. 6 (1958), 585.

4. V. R. Pandharipande, Nucl. Phys. A 178 (1971), 123.

5. B. D. DAY, Rev. Mod. Phys. 39 (1967), 719.

6. L. Shen and C. W. Woo, Phys. Rev. D 10 (1974), 371.

7. J. C. OWEn, R. F. Bishop, AND J. M. IRvine, Phys. Lett. B 59 (1975), 1.

8. M. L. Ristig, W. J. Ter Louw, and J. W. Clark, Phys. Rev. C 3 (1971), 1504.

9. V. R. Pandharipande, Nucl. Phys. A 174 (1971), 641.

10. F. Iwamoto and M. Yamada, Prog. Theor. Phys. 18 (1957), 345; J. W. Clark, P. M. Lam, AND W. J. Ter Louw, Nucl. Phys. A 255 (1975), 1.

11. V. R. Pandharipande, Nucl. Phys. A 181 (1972), 33.

12. R. V. ReI, Ann. Physics 50 (1968), 411.

13. S. Chakravarty, M. D. Miller, and C. W. Woo, Nucl. Phys. A 220 (1974), 233.

14. J. De Boer ANd A. M. Michels, Physica 5 (1938), 945.

15. D. Schiff ANd L. Verlet, Phys. Rev. 160 (1967), 208.

16. H. A. BetHe, Ann. Rev. Nucl. Sci. 21 (1971), 93.

17. J. C. OWen, R. F. Bishop, ANo J. M. Irvine, Nucl. Phys, in press. 